\title{
Determinantes Ambientais e Sociais da Esquistossomose Mansoni em Ravena, Minas Gerais, Brasil
}

\author{
Environmental and Social Determinants in Schistosomiasis Mansoni in \\ Ravena, Minas Gerais, Brazil
}

Pedro Coura-Filho ${ }^{1}$; Márcio William C. Farah ${ }^{2}$; Dilermando F. de Rezende ${ }^{3}$;
Simone da Silva Lamartine ${ }^{1}$; Omar S. Carvalho ${ }^{1}$ \& Naftale Katz ${ }^{1}$

COURA-FILHO, P.; FARAH, M. W. C.; REZENDE, F. D.; LAMARTINE, S. S.; CARVALHO, O. S. \& KATZ, N. Environmental and Social Determinants in Schistosomiasis Mansoni in Ravena, Minas Gerais, Brazil. Cad. Saúde Públ., Rio de Janeiro, 11 (2): 254-265, Apr/Jun, 1995.

This study identified the role of biological and social determinants in the transmission of schistosomiasis mansoni in Ravena, Minas Gerais, Brazil, in 1980. This data was used to characterize the clinical and epidemiological profiles of the endemic desease in the population, allowing for the determination of the efficacy of the potable water supply and the specific treatment of those infected with $S$. mansoni. The district contains three locations, Ravenopolis, Ravena and Lavapes, where the prevalence of the endemic disease was, 20.1\%, $42.6 \%$ and $63.9 \%$, respectively. The prevalence in the district was statistically higher in men. The age brackets that displayed differences by gender were 10-14 and 15-19 years. Severity of infection was statistically different among individuals within the 10-14 year bracket in ali three locations, and in the 15-19 year bracket among individuals from Ravenopolis and Ravena. The hepatointestinal form was associated with age, and individuals under 15 years of age presented risk of infection 8.85 times higher than adults. Multivariable analysis of the factors involved in transmission of the disease showed that Lavapes was independently associated with infection. In that area, poor sanitary conditions and the proximity of houses to streams infested with S. marsoni cercariae facilitated infection of neighborhood women while performing domestic activities, as well as men digging sand from the streams for construction. These results show the focal nature of transmission of the endemic requiring specific intervention for effective control of dis ease.

Key words: Schistosomiasis; Epidemiology; Water Supply; Control Measures; Sanitation

\section{INTRODUÇÃO}

A proposta de melhoria da qualidade de vida de populações passa necessariamente pelo controle de diversas endemias. Entre elas, situa-se a esquistossomose, que ocorre em 76

\footnotetext{
Centro de Pesquisas René Rachou, Fundação Oswaldo Cruz. Av. Augusto de Lima, 1715. Caixa Postal 1743, Belo Horizonte, MG, 30190-002, Brasil.

${ }^{2}$ Fundação Mineira de Arte, Universidade do Estado de Minas Gerais. Avenida Amazonas, 6252, Belo Horizonte, MG, 30530-000, Brasil.
}

${ }^{3}$ Fundação Nacional de Saúde. Avenida Brasil, 2023, Belo Horizonte, MG, 30140-002, Brasil. países, atingindo 200 milhões de pessoas. Além dessas, há cerca de 500 a 600 milhões expostas ao risco de infecção (CNPq, 1978; WHO, 1985).

Vários autores citam a esquistossomose como uma doença complexa com variados fatores causais e ampla distribuição geográfica, motivo pelo qual a inserem no rol das doenças consideradas problemas de saúde pública. As diversas formas de organização social em uma sociedade (biótopos associados ao modo de vida, lazer, cultura, trabalho, etc.) interagem numa rede de influências que definem o perfil epidemiológico da esquistossomose (Barbosa, 1968; WHO, 1985). 
Quando sociedades antrópicas se formam em biótopos anteriormente considerados silvestres, ocupando esse espaço de forma desordenada e desigual, começam a se acentuar as diferenças na qualidade de vida de seus habitantes. Isso porque nem todos têm acesso equivalente à educação, serviço de saúde, remuneração adequada do trabalho, de forma a satisfazer a reprodução social familiar, moradia, saneamento, etc. Assim sendo, os indicadores epidemilógicos variam de uma comunidade para outra (Farooq et al., 1966; Jordan, 1977; Jordan et al., 1982).

Para caracterizar o perfil clínico-epidemiológico da esquistossomose em Ravena, Minas Gerais, buscou-se estabelecer associação dos indicadores específicos da endemia (prevalência, intensidade da infecção e formas clínicas) com comportamentos da população junto a águas naturais e condições sócio-econômicas da população. O estudo objetivou ainda identificar os fatores determinantes da endemia nas localidades que compõem o distrito. Em avaliação posterior serão verificados: a) eficácia da análise dos fatores de risco na indicação de medidas de controle; b) impacto dessas medidas, se adotadas, no controle da morbidade, bem como; c) capacidade desse método de caracterizar a dinâmica de transmissão da endemia na área estudada.

\section{MATERIAL E MÉTODOS}

\section{Área do Projeto}

O distrito de Ravena situa-se no município de Sabará, a 30Km de Belo Horizonte. Depende econômica e socialmente da capital e está inserido em sua região metropolitana.

A área é composta por três localidades: Ravenópolis, Ravena e Lavapés que, em 1980, época da coleta dos dados para realização deste estudo, tinham 470, 581 e 123 habitantes, respectivamente. A região é banhada por córregos cujas águas servem às populações das localidades para recreação, atividades domésticas e profissionais, como retirada de areia e agricultura.

\section{Mapeamento e Censo Demográfico da Área}

Foi realizado mapeamento das localidades, e todas as casas foram numeradas. Cada mora- dor recebeu um número de ordem para identificação, e seus dados foram anotados: nome, sexo e data do nascimento. Moradores que trabalhavam fora e apenas dormiam na localidade foram incluídos no estudo.

\section{Coleções Hídricas e Levantamento Malacológico}

Todas as coleções hídricas existentes no perímetro urbano do distrito foram identificadas e medidas. Os cursos de água foram percorridos e submetidos a levantamentos malacológicos mensais durante seis meses (maio a outubro). Uma concha metálica perfurada era mergulhada 10 vezes na água a cada 10 passos. Os moluscos capturados eram acondicionados em sacos plásticos, identificados por localidade e transportados ao laboratório, para classificação, mensuração e exame para S. mansoni.

\section{Sentinelas Biológicos}

A exposição de camundongos-sentinelas foi realizada mensalmente em pontos das coleções hídricas, onde 10 camundongos albinos eram acondicionados individualmente em gaiolas de tela metálica com $3,5 \mathrm{~cm}$ de altura por $10 \mathrm{~cm}$ de diâmetro, munidas com tampas plásticas, fixadas em flutuadores de madeira e isopor com 50 cm de lado (Paulini \& Dias, 1971).

Os camundongos foram introduzidos na água entre 10:30h e 15h, durante uma hora por dia, por três dias consecutivos. Decorridos 45 dias da exposição, foi feita a perfusão do sistema porta dos camundongos para recuperação de vermes de $S$. mansoni (Pellegrino \& Katz, 1968).

\section{Exame Coproparasitológico}

O exame coproparasitológico da população foi realizado um mês após o censo populacional, utilizando o método Kato-Katz (Katz et al., 1972). Foi considerado positivo o indivíduo que apresentou pelo menos um ovo de $S$. mansoni em uma das duas lâminas. Quando ovos de S. mansoni eram encontrados nas duas lâminas, considerou-se a média aritmética do número de ovos por grama de fezes (opg) como indicador da intensidade da infecção no indivíduo. Para 
grupos de indivíduos e para a população geral, considerou-se a média geométrica de opg obtidos nos indivíduos.

\section{Exame Clínico}

Na classificação da forma clínica da endemia foi adotada a sugerida por Pessoa \& Barros (1953), modificada por Barbosa (1966): Tipo I: fígado e baço não palpáveis (forma intestinal); Tipo II: fígado palpável com a consistência aumentada e baço não palpável (forma hepatointestinal); Tipo III: baço palpável com a consistência normal ou aumentada (forma hepatoesplênica).

\section{Caracterização da População}

Todas as 216 casas foram pesquisadas quanto às condições sócio-econômicas dos residentes. Questionários individuais foram aplicados em amostra estratificada aleatória (21\% da população, somando 257 pessoas), por localidade, obtendo-se informações sobre o comportamento da população em relação às coleções hídricas.

\section{Análise Estatística}

No estudo das variáveis pesquisadas sobre possível associação com a prevalência da endemia na área, foram utilizados o qui-quadrado com correção de Yates e o teste exato de Fisher para comparação de proporções (Armitage, 1987; Levin, 1987).

Nas análises de variância para médias Logarítmicas, a estatística $\mathrm{F}$ foi empregada na comparação de médias em amostras paramétricas, pelo teste de Bartlett para verificar a homogeneidade da variância dos dados calculados (Armitage, 1987); a prova H de Kruslal-Wallis nas análises de variância em amostras não-paramétricas (Levin, 1987); e o teste t de Student nas comparações entre médias de duas amostras (Armitage, 1987). A identificação de diferenças significativas entre várias médias logarítmicas foi realizada pelo teste de Newman \& Keuls (Snedecor \& Cochran, 1977).

O cálculos do Odds ratio, foi usado na verificação da associação entre as variáveis dependente e independentes (Shlesselman, 1982). O método utilizado foi o de Cornfield (Armitage, 1987). Os Odds ratio foram ajustados pela análise multivariada para identificar o efeito independente de cada variável (Breslow \& Day, 1980). As variáveis incluídas no modelo logístico foram selecionadas segundo os critérios sugeridos por Greenland (1989).

Todas as variáveis testadas univariadamente foram comparadas entre si com o objetivo de selecionar aquelas a serem incluídas no modelo logístico: localidade, origem da água para tomar banho, origem da água para lavar roupa, instalações sanitárias, contato com águas naturais, ocupação principal, sexo e idade, ajustadas hierarquicamente, seguindo o valor decrescente de Odds ratio.

O nível de significância adotado no tratamento estatístico dos dados foi de $95,0 \%$.

Para o processamento estatístico dos dados em microcomputador, foram utilizadas as linguagens de programação DBase III Plus e Clipper, versão 5.01. Os pacotes estatísticos foram o Epi-Info (Dean et al., 1990) e MULTLR (Campos-Filho \& Franco, 1989).

\section{RESULTADOS}

\section{Captura de Planorbídeos}

$\mathrm{Na}$ área de Ravena, foram identificadas 15 coleções hídricas com total de $9845 \mathrm{~m}$ lineares, compostas pelos córregos Florêncio, Ravenópolis, Águas Claras, Lavapés e Corguinho e o rio Vermelho, além de quatro valas, três poços e duas pequenas lagoas.

Foram capturados 8989 planorbídeos, sendo 3812 exemplares de Biomphalaria glabrata, em 12 coleções hídricas distintas, e 5177 de Biomphalaria tenagophila, em um único curso de água.

Do total de $B$. glabrata capturadas, 58 $(1,5 \%)$ encontravam-se parasitadas por S. mansoni. No córrego Lavapés, foram encontradas $1557(40,8 \%)$ do total, e $55(3,5 \%)$ delas estavam infectadas (eliminavam cercárias de $S$. mansoni). No córrego Águas Claras, foram encontradas $1038(27,25 \%)$ espécimes de $B$. glabrata, estando $2(0,2 \%)$ infectadas. No rio Vermelho, foram encontrados $62(1,6 \%)$ exemplares de $B$. glabrata, estando somente $1(1,6 \%)$ 
infectado. Não foi observado nenhum exemplar de B. tenagophila abrigando cercárias de S. mansoni.

\section{Sentinelas Biológicos}

A taxa de camundongos infectados com $S$. mansoni variou de $1,1 \%$ a $40,8 \%$ nos sentinelas colocados no córrego Lavapés, e $0,4 \%$ a 1,2\% no rio Vermelho. Nas outras coleções hídricas, não foi observada infecção dos camundongos pelo $S$. mansoni.

\section{Estudo de Prevalência}

Do total de 1174 residentes no distrito, 998 $(85,0 \%)$ foram examinados, sendo 79,6\% (3741 470), 86,9\% (505/581) e 96,7\% (119/123) em Ravenópolis, Ravena e Lavapés, respectivamente.
A prevalência da infecção pelo $S$. mansoni nos residentes do distrito foi de $36,7 \%$. Na localidade de Ravenópolis, foi de 20,1\% em Ravena, 42,6\%, e em Lavapés, 63,9\%. Ocorreu diferença significativa da prevalência entre as localidades (Tabela 1 ).

A prevalência da esquistossomose nos indivíduos do sexo feminino no distrito foi $30,3 \%$ e, nos do sexo masculino, 42,9\%. Houve associação da infecção pelo $S$. mansoni com o sexo masculino dos infectados no distrito $\left(\mathrm{X}^{2}=16,62\right.$; $\mathrm{p}=0,0000)$ (Tabela 2).

As faixas etárias que apresentaram diferenças por sexo dos infectados foram as de 10-14 $\left(X^{2}=6,23 ; p=0,0126\right)$ e de $15-19$ anos $\left(X^{2}=4,83\right.$; $\mathrm{p}=0,0279)$. Indivíduos nas faixas 10-14 e 15-19 anos do sexo masculino tinham $2,45(1,19-5,10) \mathrm{e}$ $2,38(1,08-5,26)$ vezes o risco de infecção pelo $S$. mansoni quando comparados com as do sexo feminino (Tabela 2).

TABELA 1. Prevalência da Esquistossomose por Localidade no Distrito de Ravena segundo Faixa Etária da População em 1980

\begin{tabular}{|c|c|c|c|c|c|}
\hline Faixas Etárias & $\begin{array}{c}\text { Ravenópolis } \\
\text { I/E (\%) }\end{array}$ & $\begin{array}{l}\text { Ravena } \\
\mathrm{I} / \mathrm{E}(\%)\end{array}$ & $\begin{array}{l}\text { Lavapés } \\
\text { UB (\%) }\end{array}$ & $\mathrm{X}^{2}$ & $\mathrm{p}$ \\
\hline $0-4$ & $2 / 62(3,22)$ & $1 / 29(3,44)$ & $6 / 18(33,33)$ & 17,90 & 0,0001 \\
\hline $5-9$ & $6 / 65(9,23)$ & $21 / 63(33,33)$ & $11 / 17(64,70)$ & 24,37 & 0,0000 \\
\hline $10-14$ & $14 / 59(23,73)$ & $39 / 66(59,09)$ & $16 / 21(76,19)$ & 23,87 & 0,0000 \\
\hline $15-19$ & $17 / 39(43,59)$ & $56 / 80(70,00)$ & $14 / 16(87,50)$ & 12,19 & 0,0023 \\
\hline $20-29$ & $14 / 47(29,78)$ & $53 / 105(50,48)$ & $10 / 15(66,67)$ & 8,40 & $\mathbf{0 , 0 1 5 0}$ \\
\hline$\geq 30$ & $22 / 101(21,78)$ & $45 / 162(27,78)$ & $19 / 32(59,37)$ & 16,95 & 0,0002 \\
\hline Total & $75 / 373(20,05)$ & $215 / 505(42,57)$ & $76 / 119(63,86)$ & 89,94 & $\mathbf{0 , 0 0 0 0}$ \\
\hline $\mathrm{p}=$ & 0,0000 & 0,0000 & 0,0070 & & \\
\hline
\end{tabular}

$\mathrm{I} / \mathrm{E}=$ infectados pelo $S$. mansoni examinados

Em negrito: valores significativos de $X^{2}$.

TABELA 2. Prevalência na População do Distrito de Ravena segundo Sexo e Faixa Etária em 1980

\begin{tabular}{lrrrrr}
\hline \hline & \multicolumn{1}{c}{$\begin{array}{l}\text { Mulheres } \\
\text { Faixa Etária }\end{array}$} & \multicolumn{1}{c}{ Homens } & \multicolumn{1}{c}{ Total } \\
I/E* $(\%)$ & \multicolumn{1}{c}{ I/E $(\%)$} & \multicolumn{1}{c}{ I/E $(\%)$} & \multicolumn{1}{c}{$X^{2}$} & \multicolumn{1}{c}{ p } \\
\hline $0-4$ & $3 / 54(5,66)$ & $6 / 56(10,71)$ & $9 / 110(8,25)$ & 0,28 & 0,4901 \\
$5-9$ & $18 / 70(25,71)$ & $20 / 75(26,67)$ & $38 / 145(26,21)$ & 0,00 & 0,9532 \\
$10-14$ & $26 / 72(36,11)$ & $43 / 74(58,11)$ & $69 / 146(47,26)$ & $\mathbf{6 , 2 3}$ & $\mathbf{0 , 0 1 2 6}$ \\
$15-19$ & $4 / 63(534,00)$ & $53 / 72(73,61)$ & $87 / 135(64,44)$ & $\mathbf{4 , 8 3}$ & $\mathbf{0 , 0 2 7 9}$ \\
$20-29$ & $29 / 77(37,66)$ & $48 / 90(53,33)$ & $77 / 167(46,11)$ & 3,49 & 0,0615 \\
$\geq 30$ & $40 / 160(25,00)$ & $46 / 135(34,10)$ & $86 / 295(29,15)$ & 2,50 & 0,1141 \\
\hline Total & $150 / 496(30,30)$ & $216 / 502(42,94)$ & $366 / 998(36,67)$ & $\mathbf{1 6 , 6 2}$ & $\mathbf{0 , 0 0 0 0}$ \\
$\mathrm{p}=$ & $\mathbf{0 , 0 0 0 0}$ & $\mathbf{0 , 0 0 0 0}$ & & \\
\hline \hline
\end{tabular}

$\mathrm{I} / \mathrm{E}=$ infectados pelo $S$. mansoni examinados.

Em negrito: valores significativos de $\mathrm{X}^{2}$ 


\section{Estudo da Intensidade da Infecção}

No distrito, a intensidade da infecção medida pela média geométrica do número de ovos por grama de fezes (opg) não estava associada ao sexo dos infectados mesmo quando ajustados por idade. Considerando-se isoladamente as mulheres e os homens, houve associação entre intensidade da infecção e faixa etária (Tabela 3).

Como pode ser visto na Tabela 4 , as médias geométricas do número de ovos de $S$. mansoni eliminados nas fezes foram significativamente diferentes entre os indivíduos das três localidades. Os moradores de Ravenópolis apresentaram média de opg menor do que a dos moradores de Ravena e Lavapés ( $\mathrm{p}=0,0000)$. Os grupos etários das localidades que apresentaram médias de opg estatísticamente diferentes foram o de 10-14 anos ( $\mathrm{p}=0,0041)$ e o de 15-19 anos ( $\mathrm{p}=0,0204)$, em Ravenópolis e Ravena (Tabela 4).

O grupo etário de Ravena que apresentou diferença da média de opg com o grupo correspondente de Lavapés foi o de 10-14 anos (F= $11,765 ; p=0,0022)$ (Tabela 4).

TABELA 3. Intensidade da Infecção pelo $S$. mansoni nas Populações das Três Localidades de Ravena, Minas Gerais, segundo o Sexo e Faixa Etária da População em 1980

\begin{tabular}{|c|c|c|c|c|}
\hline Faixa Etária & $\begin{array}{l}\text { Mulheres } \\
\text { (N) G } \pm s^{*}\end{array}$ & $\begin{array}{l}\text { Homens } \\
\text { (N) G } \pm s^{*}\end{array}$ & $\begin{array}{c}\text { Total } \\
\text { (N) G } \pm \mathrm{s}^{*}\end{array}$ & $\mathrm{P}$ \\
\hline $0-4$ & (3) $85,4 \pm 3,18$ & (6) $112,7 \pm 2,57$ & (9) $102,7 \pm 2,58$ & 0,5249 \\
\hline $5-9$ & (18) $206,0 \pm 4,61$ & (20) $276,8 \pm 3,73$ & (38) $253,4 \pm 4,17$ & 0,6699 \\
\hline $10-14$ & (26) $322,2 \pm 3,83$ & (43) $368,2 \pm 3,14$ & (69) $350,2 \pm 3,37$ & 0,8208 \\
\hline $15-19$ & (34) $233,4 \pm 3,40$ & (53) $343,0 \pm 3,24$ & (87) $295,1 \pm 3,33$ & 0,5951 \\
\hline $20-29$ & (29) $174,2 \pm 6,11$ & (48) $237,1 \pm 4,04$ & (77) $211,3 \pm 4,75$ & 0,5898 \\
\hline$\geq 30$ & (40) $137,2 \pm 3,07$ & (46) $118,4 \pm 2,92$ & (86) $126,8 \pm 3,00$ & 0,2948 \\
\hline \multirow[t]{3}{*}{ Total } & (150) $200,0 \pm 3,75$ & (216) $251,7 \pm 3,58$ & (366) $228,9 \pm 3,66$ & 0,2170 \\
\hline & $H=40,678$ & $F=6,378$ & & \\
\hline & $P=0,0000$ & $P=0,0000$ & & \\
\hline
\end{tabular}

* média geométrica do número de ovos de S.mansoni por grama de fezes, \pm desvio padrão.

Em negrito: valores significativos de ANOVA.

TABELA 4. Intensidade da Infecção pelo Schistosoma mansoni na População do Distrito de Ravena, segundo a Faixa Etária, em 1980

\begin{tabular}{|c|c|c|c|c|}
\hline Faixa Etária & $\begin{array}{c}\text { Ravenópolis } \\
(\mathrm{N}) \mathrm{G} \pm \mathrm{s}\end{array}$ & $\begin{array}{l}\text { Ravena } \\
(\mathrm{N}) \mathrm{G} \pm \mathrm{s}\end{array}$ & $\begin{array}{l}\text { Lavapés } \\
(\mathrm{N}) \mathrm{G} \pm \mathrm{s}\end{array}$ & $\mathrm{p}$ \\
\hline $0-4$ & (2) $160,1 \pm 1,52$ & (1) 24,0 & (6) $112,7 \pm 2,57$ & 0,3323 \\
\hline $5-9$ & (6) $448,0 \pm 4,08$ & (21) $226,7 \pm 4,29$ & (11) $229,5 \pm 4,23$ & 0,8124 \\
\hline $10-14^{*}$ & (14) $189,6 \pm 2,74$ & (39) $311,5 \pm 3,35$ & (16) $796,4 \pm 2,73$ & 0,0041 \\
\hline $15-19 * *$ & (17) $172,3 \pm 3,77$ & (56) $358,8 \pm 3,13$ & (14) $259,3 \pm 3,17$ & 0,0710 \\
\hline $20-29$ & (14) $139,0 \pm 5,89$ & (53) $209,4 \pm 4,61$ & (10) $398,1 \pm 5,00$ & 0,2665 \\
\hline$\geq 30$ & (22) $93,8 \pm 1,85$ & (45) $138,7 \pm 3,02$ & (19) $145,5 \pm 4,24$ & 0,8231 \\
\hline \multirow[t]{3}{*}{ Total } & (75) $158,8 \pm 3,16$ & (215) $244,0 \pm 3,55$ & (76) $281,8 \pm 3,98$ & 0,0127 \\
\hline & $\mathbf{H}=32,291$ & $F=5,483$ & $F=3,612$ & \\
\hline & $P=0,0000$ & $\mathbf{p}=\mathbf{0 , 0 0 0 2}$ & $p=0,0080$ & \\
\hline
\end{tabular}

$\mathrm{G} \pm \mathrm{s}=$ média geométrica do número de ovos de $S$. mansoni por grama de fezes, \pm desvio padrão.

Em negrito: valores significativos de ANOVA.

* Faixa etária cuja média de ovos de S. mansoni foi estatisticamente diferente entre os moradores das três localidades. Entre Ravena e Lavapés, F = 11,77 ( $\mathrm{p}=0,0022$ ).

** Faixa etária cuja média de ovos de $S$. mansoni foi estatisticamente diferente entre os moradores de Ravenópolis e Ravena $(\mathrm{p}=0,0204)$. 


\section{Estudo das Formas Clínicas}

Os exames clínicos para a esquistossomose na população do distrito de Ravena revelaram ocorrência apenas das formas intestinais e hepatointestinais. Houve significativa diferença das formas clínicas entre crianças e adultos. $\mathrm{O}$ risco de adquirirem a forma hepatointestinal foi $8,85(3,31$ - 27,36) vezes maior em crianças do que nos adultos. As formas clínicas não estive- ram associadas ao sexo, cor e intensidade de infecção (Tabela 5).

\section{Análise Multivariada}

Ao final dos ajustamentos na análise multivariada, a variável que se manteve associada independentemente à infecção foi o fato de o indivíduo morar na localidade de Lavapés (Tabela 6, 7 e 8).

TABELA 5. Formas Clínicas da Esquistossomose Mansoni segundo Variáveis Selecionadas, no Distrito de Ravena, em 1980

\begin{tabular}{|c|c|c|c|c|}
\hline \multirow[b]{2}{*}{ Variáveis } & \multicolumn{2}{|c|}{ Formas Clínicas } & \multirow[b]{2}{*}{$\mathrm{p}$} & \multirow[b]{2}{*}{ Odds Ratio } \\
\hline & $\begin{array}{c}\text { Hepatointestinal } \\
\mathrm{n}(\%)\end{array}$ & $\begin{array}{c}\text { Intestinal } \\
\mathrm{n}(\%)\end{array}$ & & \\
\hline \multicolumn{5}{|l|}{ a. Localidades } \\
\hline Ravenópolis & $4(15,38)$ & $22(84,62)$ & & 1,00 \\
\hline Ravena & $19(14,96)$ & $108(85,04)$ & & $1,97(0,27-3,74)$ \\
\hline Lavapés & $11(22,45)$ & $38(77,55)$ & 0,4816 & $1,59(0,40-6,81)$ \\
\hline \multicolumn{5}{|l|}{ b. Idade } \\
\hline$\leq 14$ anos & $28(82,35)$ & $58(34,52)$ & & $8,85(3,31-27,36)$ \\
\hline$\geq 15$ anos & $06(17,65)$ & $110(65,48)$ & 0,0000 & 1,00 \\
\hline \multicolumn{5}{|c|}{ c. Intensidade da Infecção } \\
\hline $12-500$ opg & $21(61,76)$ & $107(64,07)$ & & 1,00 \\
\hline$\geq 501$ opg & $13(38,24)$ & $60(35,93)$ & 0,9526 & $1,10(0,48-2,51)$ \\
\hline \multicolumn{5}{|l|}{ d. Sexo } \\
\hline Mulheres & $13(38,67)$ & $72(42,86)$ & & 1,00 \\
\hline Homens & $21(61,76)$ & $96(57,14)$ & 0,7585 & $1,21(0,54-2,76)$ \\
\hline \multicolumn{5}{|l|}{ e. Cor da Pele } \\
\hline Negra & $7(12,73)$ & $28(7,80)$ & & $1,72(0,65-4,43)$ \\
\hline Não negra & $48(87,27)$ & $331(92,20)$ & 0,2931 & 1,00 \\
\hline f. $(\mathrm{N}) \mathrm{G} \pm \mathrm{s}$ & (34) $244,5 \pm 4,40$ & (168) $264,7 \pm 3,67$ & $0,5055^{*}$ & \\
\hline
\end{tabular}

* Teste $\mathrm{t}$ de STUDENT.

TABELA 6. Fator, Determinado na Análise Multivariada, Associado à Infecção pelo Schistosoma mansoni na População do Distrito de Ravena em 1980

\begin{tabular}{|c|c|c|c|c|}
\hline & Positivos & Negativos & $\mathrm{p}$ & \\
\hline Localidade & $\mathrm{n}\left(\%^{\circ}\right)$ & $\mathrm{n}(\%)$ & (log-likelihood) & Odds Ratio* \\
\hline - Lavapés & $76(63,87)$ & $43(36,13)$ & & $7,07(1,17-42,67)$ \\
\hline • Não Lavapés & $290(32,99)$ & $589(67,01)$ & 0,0328 & 1,00 \\
\hline
\end{tabular}

* Odds Ratio ajustado pela regressão logística. 
TABELA 7. Variáveis Associadas à Infecção pelo S. mansoni, segundo a Localidade do Distrito de Ravena, em 1980

\begin{tabular}{|c|c|c|c|c|c|c|c|c|}
\hline \multirow[b]{2}{*}{ Variáveis } & \multicolumn{2}{|c|}{$\begin{array}{c}(1) \\
\text { Ravenópolis }\end{array}$} & \multicolumn{2}{|c|}{$\begin{array}{c}(2) \\
\text { Ravena }\end{array}$} & \multicolumn{2}{|c|}{$\begin{array}{c}(3) \\
\text { Lavapés }\end{array}$} & \multirow[b]{2}{*}{$\mathrm{p}$} & \multirow[b]{2}{*}{ Odds ratio $(\mathrm{OR})$} \\
\hline & + & - & + & - & + & - & & \\
\hline $\begin{array}{l}\text { - Ter contato com águas } \\
\text { naturais }\end{array}$ & 11 & 36 & 31 & 29 & 17 & 8 & 0,0001 & $\begin{array}{l}\text { (1) } 1.00 \\
\text { (2) } 3,50(1,40-8,91) \\
\text { (3) } 6.95(2,10-23,90)\end{array}$ \\
\hline $\begin{array}{l}\text { - Ausência de água de cisterna } \\
\text { para beber }\end{array}$ & 5 & 14 & 43 & 64 & 13 & 6 & 0,0088 & $\begin{array}{l}\text { (1) } 1,00 \\
\text { (2) } 1,88(0,57-6,50) \\
\text { (3) } 6,07(1,23-32,64) \\
\end{array}$ \\
\hline $\begin{array}{l}\text { - Ausência de água de cisterna } \\
\text { para lavar vasilhas }\end{array}$ & 5 & 13 & 43 & 64 & 13 & 6 & 0,0088 & $\begin{array}{l}\text { (1) } 1,00 \\
\text { (2) } 1,75(0,53-6,10) \\
\text { (3) } 5,63(1,13-30,58) \\
\end{array}$ \\
\hline $\begin{array}{l}\text { - Ausência de água de cisterna } \\
\text { para lavar roupas }\end{array}$ & 8 & 42 & 43 & 64 & 13 & 6 & 0,0000 & $\begin{array}{l}\text { (1) } 1,00 \\
\text { (2) } 3,53(1,42-9,05) \\
\text { (3) } 11,38(3,14-51,00) \\
\end{array}$ \\
\hline $\begin{array}{l}\text { - Ausência de água de cisterna } \\
\text { para tomar banho }\end{array}$ & 5 & 12 & 43 & 64 & 14 & 7 & 0,0171 & $\begin{array}{l}\text { (1) } 1,00 \\
\text { (2) } 1,61(0,48-5,70) \\
\text { (3) } 4,80(1,01-24,78) \\
\end{array}$ \\
\hline - Frequiência semanal de contato & 6 & 22 & 20 & 27 & 12 & 3 & 0,0003 & $\begin{array}{l}\text { (1) } 1,00 \\
\text { (2) } 2,72(0,83-9,16) \\
\text { (3) } 14,67(2,56-97,73)\end{array}$ \\
\hline - Instalações sanitárias & 4 & 13 & 29 & 44 & 13 & 6 & 0,0085 & $\begin{array}{l}\text { (1) } 1,00 \\
\text { (2) } 1,98(052-8,12) \\
\text { (3) } 6,50(1,31-42,03) \\
\end{array}$ \\
\hline - Sexo masculino & 49 & 123 & 84 & 150 & 36 & 40 & 0,0043 & $\begin{array}{l}\text { (1) } 1,00 \\
\text { (2) } 1,41(0,90-2,20) \\
\text { (3) } 2,26(1,24-4,11)\end{array}$ \\
\hline • Não trabalha & 9 & 40 & 19 & 31 & 8 & 4 & 0,0088 & $\begin{array}{l}\text { (1) } 1,00 \\
\text { (2) } 7,05(2,80-18,11) \\
\text { (3) } 8,89(1.84-46,41) \\
\end{array}$ \\
\hline - Ter propriedade da casa & IS & 40 & 44 & 50 & 15 & 7 & 0,0006 & $\begin{array}{l}\text { (1) } 1,00 \\
\text { (2) } 2,35(1,08-5,14) \\
\text { (3) } 5,71(1,74-19,46)\end{array}$ \\
\hline
\end{tabular}

+ positivo para a infeção pelo S. mamoni.

- negativo para a infecção.

TABELA 8. Resultados da Regressão Logística Múltipla das Variáveis de Confusão no Distrito de Ravena em 1980

\begin{tabular}{lcc}
\hline \hline Variáveis & $\mathrm{p}$ & Odds Ratio \\
\hline a. Ravenópolis & Baseline & \\
b. Ravena & 0,7619 & \\
c. Lavapés & 0,0328 & $7,07(1,17-42,667)$ \\
d. Origem da água para tomar banhos & 0,5713 & \\
e. Origem da água para lavar roupas & 0,7312 & \\
f. Tipo de instalações sanitárias & 0,4043 & \\
g. Ter contato com águas naturais & 0,3582 & \\
h. Tipo de ocupação (estudante e dona-de-casa) & 0,3573 & \\
i. Sexo & 0,2727 & \\
j. Idade: adultos & 0,1844 & \\
\multicolumn{1}{c}{ crianças } & 0,0541 & \\
\hline \hline
\end{tabular}

Em negrito: valor significativo de p. 
O fato de a variável ocupação ("estudante", "donas-de-casa" e "não trabalha") ter sido eliminada com o ajustamento do sexo ao modelo logístico - denota forte covariância entre elas no que se refere a riscos de infecção pelo $S$. mansoni, em Ravenópolis e Ravena. Esse resultado esteve de acordo com os maiores riscos de infecção observados entre os homens $(\mathrm{OR}=1,74)$ e entre os indivíduos que não trabalham $(\mathrm{OR}=2,04)$, que, em sua maioria, são estudantes e donas-de-casa. Aliados a esses resultados estão os maiores riscos de contato com águas naturais observados, em estudantes, para nadar e, em donas-de-casa, para lavar roupas (Tabela 9).

\section{DISCUSSÃO}

A infecção mais intensa observada em sentinelas biológicos colocados em coleções hídricas com baixa velocidade do fluxo em águas próximas de Lavapés contribuiu para os maiores índices de infecção pelo $S$. mansoni. No córrego Lavapés, que corta a localidade de mesmo nome, a infecção em sentinelas biológicos chegou a 40,8\%. Nesse córrego, 3,5\% dos exemplares de B. glabrata capturados eliminavam cercárias do parasita. No rio Vermelho, os índices variaram entre $0,4 \%$ e $1,2 \%$. Esse rio, mais distante das casas e de fluxo mais rápido, forneceu apenas um molusco eliminando cercárias.

TABELA 9. Comparação das Formas de Contatos com Água entre Indivíduos de Ambos os Sexos no Distrito de Ravena em 1980

\begin{tabular}{|c|c|c|c|c|c|}
\hline Variáveis & Homens & Mulheres & $\mathrm{p}$ & Odds Ratio & $\begin{array}{l}\text { Percentual de } \\
\text { Freqüência do Contato }\end{array}$ \\
\hline \multicolumn{6}{|l|}{ a. Nadar } \\
\hline Sim & 81 & 57 & & $3,02(1,91-6,61)$ & $53,70 \%$ \\
\hline Não & 48 & 10 & 0,0059 & 1,00 & \\
\hline \multicolumn{6}{|c|}{ b. Lavar roupa } \\
\hline Sim & 0 & 56 & & Indefinido* & $21,79 \%$ \\
\hline Nilo & 128 & 72 & 0,0000 & & \\
\hline \multicolumn{6}{|c|}{ c. Tomar banho } \\
\hline Sim & 33 & 8 & & $1,55(1,20-2,95)$ & $15,95 \%$ \\
\hline Não & 96 & 120 & 0,0000 & 1,00 & \\
\hline \multicolumn{6}{|l|}{ d. Pescar } \\
\hline Sim & 24 & 2 & & $1,17(1,02-2,09)$ & $10,12 \%$ \\
\hline Não & 105 & 125 & 0,0000 & 1,00 & \\
\hline \multicolumn{6}{|c|}{ e. Atravessar córregos } \\
\hline Sim & 9 & 10 & & & $7,39 \%$ \\
\hline Não & 117 & 116 & 1,0000 & & \\
\hline \multicolumn{6}{|c|}{ f. Lavar vasilhas } \\
\hline Sim & 0 & 14 & & Indefinido* & $5,44 \%$ \\
\hline Não & 129 & 114 & 0,0000 & & \\
\hline \multicolumn{6}{|c|}{ g. Explorar minério (areia) } \\
\hline Sim & 8 & 0 & & Indefinido* & $3,11 \%$ \\
\hline Não & 121 & 127 & 0,070 & & \\
\hline \multicolumn{6}{|c|}{ h. Trabalhar na lavoura } \\
\hline Sim & 3 & 1 & & & $1,56 \%$ \\
\hline Não & 126 & 126 & 1,0000 & & \\
\hline \multicolumn{6}{|c|}{ i. Regar horta } \\
\hline Sim & 1 & 0 & & & $0,38 \%$ \\
\hline Não & 128 & 127 & 1,0000 & & \\
\hline
\end{tabular}


A prevalência geral no distrito para a infecção pelo S. mansoni foi de $36,7 \%$. O aumento desse índice até a segunda década de vida foi observado na maioria das áreas endêmicas descritas na literatura, o que pode ser devido tanto à intensidade e ao tipo de contato com águas naturais estabelecidos pelos diversos grupos etários quanto à imunidade adquirida com o aumento da idade dos indivíduos expostos (Katz et al., 1978).

A prevalência e a média geométrica do número de opg apresentaram correlação positiva entre si, mesmo nos grupos etários mais velhos. Essa correlação foi também observada por Lehman Jr. et al., 1976; Lima e Costa et al., 1986; Kloetzel \& Shuster, 1987; Kloetzel \& Vergetti, 1988; Barreto, 1991; entre outros, nas áreas por eles estudadas.

As altas médias de opg e os baixos desvios-padrão observados nos grupos etários 10-14 e 15-19 anos de idade - indicam que eles são os grupos mais homogêneos. Segundo Katz \& Rocha (1991) só ocorre diferença na infecção, por sexo, quando há fatores causais profissionais ou culturais.

A prevalência da infecção na área foi maior nos homens com idade entre 10-19 anos quando comparada com a de mulheres da mesma idade. Os maiores motivos de contatos com águas naturais pelos homens dessa idade foram: nadar $\mathrm{OR}=3,02$; tomar banho $\mathrm{OR}=1,55 \mathrm{e}$ pescar $\mathrm{OR}=1,17$.

A forma de reprodução social no distrito de Ravena, mais do que a forma de produção, definiu o perfil clínico-epidemiológico da endemia. Sessenta e um por cento das contagens acima de 500 opg ocorreram em homens que tiveram contatos semanais com águas naturais para natação e banho. Embora a maior parte dos moradores do distrito com contagens de ovos acima de 500 opg fosse de trabalhadores rurais $(28,0 \%)$, os contatos não eram por motivos profissionais e não levaram a formas graves da esquistossomose. Isso porque o trabalho agrícola comum na área (plantações de banana e pequenas lavouras de milho, café e hortaliças) requer poucos contatos com águas naturais.

Crianças até 14 anos de idade apresentaram as maiores médias de opg no distrito e apresentaram também maior risco de adquirir forma clíni- ca hepatointestinal quando comparadas com os adultos. Também Santos \& Coura (1986), em Padre Paraíso, Minas Gerais, encontraram o maior percentual de casos de formas hepatointestinais em crianças entre 6-15 anos; Lehman Jr. et al. (1976), em Castro Alves, Bahia, em crianças entre 10-14 anos; Guerra (1992), em São José do Acácio, Minas Gerais, em crianças com até 14 anos de idade.

Os padrões de contatos com águas naturais são estabelecidos de maneira diferente entre grupos de moradores das localidades em função da desigualdade na distribuição da água potável, sendo que Lavapés não possuía uma só casa abastecida com água tratada. As instalações sanitárias e a origem da água, para beber, lavar vasilhas, lavar roupas e tomar banhos, eram significativamente diferentes nas localidades. Lavapés sempre apresentou piores condições sanitárias em relação a Ravenópolis e Ravena. Em Lavapés, a prevalência entre os residentes chegou a $63,86 \%$, e $46,34 \%$ dos casos apresentaram carga parasitária acima de 500 opg.

Estudos realizados por Farooq \& Mallah (1966), Dalton (1976), Guimarães et al. (1985) Kvalsvig \& Shutte (1986) e Chandiwana \& Woohouse (1991), Lima e Costa et al.(1991) e Guerra (1992) analisaram áreas onde contatos com água naturais ocorriam por lazer e em atividades domésticas. Uma análise sobre o uso do paradigma de risco para a esquistossomose em sete áreas endêmicas sugere maior tendência de indivíduos de áreas rurais terem em torno de $70 \%$ dos contatos com águas naturais em atividades profissionais (agricultura e domésticas); nas áreas mais urbanizadas, esse percentual é observado por motivo de lazer (Coura-Filho, 1994).

Ravenópolis, localidade montanhosa, com menor prevalência e intensidade de infecção, foi a localidade cujos moradores tinham menos contatos com águas infestadas pelo $S$. manso$n i$. A principal fonte de água nessa localidade era cisterna, que capta água de lençóis freáticos, sem chances de terem caramujos com cercárias de S. mansoni. Em 83,50\% dos domicílios havia fossa, o que reduz a contaminação peridomiciliar, forma freqüente de transmissão da esquistossomose em diversas áreas. 
Ravena, embora com melhores condições de saneamento do que Ravenópolis e Lavapés, carecia de opções de lazer. Campos de futebol próximos ao córrego Lavapés e ao rio Vermelho (ao lado de um ponto de escoamento de esgoto de Ravena), favoreciam contatos com águas naturais.

Embora morar em Lavapés tenha sido a única associação independente à esquistossomose, esse resultado não invalida a análise univariada feita anteriormente. Indica que os riscos de infecção pelo $S$. mansoni no distrito de Ravena são causados pela ação conjunta das variáveis analisadas. Tomada isoladamente uma delas (exceto a variável "morar em Lavapés"), as outras não podem ser consideradas causas da infecção. Das associações univariadas verificadas no distrito foram identificadas oito variáveis de confusão (Greenland, 1989): localidade, contato com águas naturais, origem da água de tomar banho, origem da água de lavar roupas, tipo de instalações sanitárias, tipo de ocupação, sexo e idade.

Os dados deste estudo reforçam a idéia de que os projetos de investimentos no setor urbano no distrito de Ravena devem priorizar a localidade de Lavapés, como forma de controle da esquistossomose na área. A eliminação dos contatos dos moradores dessa localidade com águas peridomiciliares, para atividades domésticas, lazer de crianças e trabalho das donas-de-casa, pode resultar em significativa redução dos indicadores epidemiológicos da esquistossomose.

A priori, para o controle da transmissão e da morbidade da doença no distrito de Ravena, o uso de mosluscicida teria função auxiliar. Mas, considerado o fato de que os focos de transmissão da esquistossomose no distrito ocorrem em córregos e rios pequenos, a maioria com forte vasão, o moluscicida seria levado pela correnteza e teria breve ação no controle da transmissão do $S$. mansoni. Devem ser considerados também os altos custos desses produtos, os danos provocados por freqüentes aplicações à biota aquática, além da questionável eficácia para reduzir de forma duradouera o número de molusco (Coura-Filho, et al., 1992).
Cabe ao saneamento básico, principalmente em Lavapés, à criação de alternativas de lazer que evitem o contato dos indivíduos com águas naturais contaminadas pelo $S$. mansoni e ao tratamento específico dos infectados o papel principal no controle da esquistossomose no distrito de Ravena (Sabará, Minas Gerais).

\section{RESUMO}

COURA-FILHO, P.; FARAH, M. W. C.; REZENDE, F. D.; LAMARTINE, S. S.; CARVALHO, O. S. \& KATZ, N. Determinantes Ambientais e Sociais da Esquistossomose Mansoni em Ravena, Minas Gerais, Brasil. Cad. Saúde Públ., Rio de Janeiro, 11 (2): 254-265, abr/jun, 1995.

Neste estudo foram identificados os determinantes biológicos e sociais na transmissão da esquistossomose em Ravena, Sabará, Minas Gerais, Brasil, em 1980, visando a caracterizar o perfil clínico-epidemiológico da endemia na população para posterior avaliação da eficácia do fornecimento de água potável intradomiciliar e o tratamento específico quadrianual dos infectados pelo Schistosoma mansoni. O distrito é formado por três localidades: Ravenópolis, Ravena e Lavapés, cujas prevalências da endemia foram $20,1 \%$; $42,6 \%$ e $63,9 \%$, respectivamente. A prevalência da endemia no distrito foi estatisticamente maior nos homens. As faixas etárias que apresentaram diferenças por sexo foram as de 10-14 e 15-19 anos. A intensidade da infecção só foi diferente estatisticamente entre indivíduos com idade entre 10 e 14 anos nas três localidades, e de 15 a 19 anos entre indivíduos de Ravenópolis e Ravena. A forma hepatointestinal estava associada à idade: menores de 15 anos apresentaram risco 8,85 vezes maior do que os adultos. A análise multivariada dos fatores determinantes da transmissão da endemia evidenciou que a localidade de Lavapés esteve independentemente associada à infecção pelo S. mansoni. Era onde estavam ocorrendo os maiores riscos de infecção por falta de saneamento, maior proximidade das casas a 
córregos infestados por cercárias de $S$. mansoni, o que facilitava a infeccção das donas-de-casa em atividades domésticas e dos homens na prática de tirar areia. Esses resultados apontam o carater focal da transmissão da endemia, exigindo medidas específicas.

Palavras-Chave: Esquistossomose; Epidemiologia; Suprimento de Água; Medidas de Controle; Saneamento

\section{REFERÊNCIAS BIBLIOGRÁFICAS}

ARMITAGE, P., 1987. Statistical Methods in Medical Research. 2nd, London: Blackwell Scientific Publications.

BARBOSA, F. S., 1966. Morbidade da esquistossomose. Revista Brasileira de Malariologia e Doenças Tropicais, (n⿳⺈ especial): 3-159.

1968. Esquistossomose mansonica: repercussões sobre a comunidade. Revista da Sociedade Brasileira de Medicina Tropical, 2: 153-156.

BARRETO, M. L., 1991. Geographical and socioeconomic fators relating to the distribuition of Schistosoma mansoni infection in an urban area of northeast Brasil. Bulletin of the World Health Organization, 69: 93-102.

BRESLOW, N. E. \& DAY, N. E., 1980. Statistical Methods in Cancer Research. The Analysis of Case Control Studies. Lyon: International Agency Research on Cancer.

CAMPOS-FILHO, N. B. S. \& FRANCO, E. L., 1989. A microcomputer program for multiple logistic regression by unconditional and conditional maximum likelihood methods. American Journal of Epidemiology, 129: 439-444.

CHANDIWANA, S. K. \& WOOLHOUSE, J. E. M., 1991. Heterogeneities in water contact patterns and the epidemiology of Schistosoma haematobium. Parasitolgy, 103: 363-370.

CNPq (Conselho Nacional de Desenvolvimento Científico e Tecnológico), 1978. Programa do Trópico Semi-Árido. Epidemiologia e Controle da Esquistossomose e o Nordeste Semi-árido. Brasília, DF: CNPq.

COURA-FILHO, P., 1994. Uso do paradigma de risco para a esquistossomose em áreas endêmicas no Brasil. Cadernos de Saúde Pública, 10: 464-472.

COURA-FILHO, P.; MENDES, N. M.; SOUZA, C. P. \& PEREIRA, J. P., 1992. The prolonged use of niclosamide as a molluscicide for the control of Schistosoma mansoni. Revista do Instituto de Medicina Tropical de São Paulo, 34: 427-431.

DALTON, G. R., 1976. A socioecological approach to the control of Schistosoma mansoni in St. Lucia. Bulletin of the World Health Organization, 54: 587-595.

DEAN, A. G.; DEAN, J. A.; BURTON, A. H. \& DIKER, R. C., 1990. Epi Info Version 5: a Word Processing, Database, and Statistics Program for Epidemiology on Microcomputers. Atlanta: Centros de Lucha contra las Enfermidades.

FAROOQ, M. \& MALLAH, M. B., 1966. The behavioural pattern or social and religious projects area. Bulletin of the World Health Organization, 35: 365-377.

FAROOQ, M.; NIELSEN, J.; SAMAAN, S. A.; MALLAH, M. B. \& ALLAM, A. A., 1966. The Epidemiology of Schistosoma haematobium and S. mansoni infections in the Egypt-49 Project Area. 3 - Prevalence of bilharziasis in relation To certain environmental factors. Bulletin of the World Health Organization, 35: 319-330.

GREENLAND, S., 1989. Modeling and variable selection in epidemiologic analysis. American Journal of Public Health, 79: 340-349.

GUERRA, H., 1992. Epidemiologia da Esquistossomose Mansoni em São José do Acácio, Minas Gerais. Análise Multivariada dos Fatores Associados à Infecção. Variáveis Sócio-Demográficas e Contatos com Águas Naturais. Tese de Mestrado, Belo Horizonte: Universidade Federal de Minas Gerais.

GUIMARÃES, M. D. C.; COSTA, M. F. F. L.; LIMA, L. B. \& MOREIRA, M. A., 1985. Estudo clinico epidemiológico da esquistossomose em escolares da Ilha, município de Arcos, Minas Gerais, Brasil. Revista de Saúde Pública, 19: 08-17.

JORDAN, P., 1977. Epidemiology and control of schistosomiasis. American Journal of Tropical Medicine \& Hygiene, 28: 55-59.

JORDAN, P.; UNRAU, G. O.; BARTHOLOMEW, R. K.; COOK, J. A. \& GRIST, E. 1982. Value of individual household water supplies in the maintenance phase of a schistosomiasis control programme in Saint Lucia, after chemotherapy. Bulletin of the World Health Organization, 52: 09-20.

KATZ, N.; CHAVES, A.; PELLEGRINO, J., 1972. A simple device for quantitative stool thicks-mear technnique in schistosomiasis mansoni. Revista do Instituto de Medicina Tropical de São Paulo, 14: 397-400. 
KATZ, N.; ZICKER, F.; ROCHA, R. S.; OLIVEIRA, V. B., 1978. Re-infection of patients in schistosomiasis mansoni endemic areas after specific treatment. I. Influence of age and worn burden. Revista do Instituto de Medicina Tropical de São Paulo, 20: 273-314.

KATZ, N. \& ROCHA, R. S., 1991. Esquistosomiasis. In: Parasitologia Clínica (A. Atias, org), pp. 319-330, Santiago: A. Atias.

KLOETZEL, K. \& SCHUSTER, H. N., 1987. Repeated mass treatment of schistosomiases mansoni: experience in hyperendemic arcas of Brazil. Transactions of Royal Society and Tropical Medicine \& Hygiene, 81: 361-370.

KLOETZEL, K. \& VERGETti, A. A., 1988. Repeated mass treatment of schistosomiasis mansoni: experience in hyperendemic arcas of Brasil. II micro-level evaluation of results. Annals of Tropical Medicine and Parasitology, 4: 367-376.

KVALSVIG, S. D. \& SHUTTE, C. H. J., 1986. The role of human water contact patters in the transmission of schistosomiasis in an informal settlement near a major industrial area. Tropical Medicine and Parasitology, 80: 13-26.

LEHMAN Jr., J. S.; MOTT, K. E.; MORROW Jr., R. H.; MUNIZ, T. M. \& BOYER, M. H., 1976. The intensity and effects of infection with Schistosoma mansoni in a rural community in Northeast Brazil. American Journal of Tropical Medicine \& Hygiene, 25: 285-294.

LEVIN, J., 1987. Estatística Aplicada às Ciências Humanas. São Paulo: Harper \& Row do Brasil.

LIMA E COSTA, M. F. F.; ROCHA, R. S. \& KATZ, N., 1986. Splenomegaly in relation to Schistosoma mansoni egg count: a population based study. Revista do Instituto de Medicina Tropical de São Paulo, 28: 389-394.
LIMA E COSTA, M. F. F.; ROCHA, R. S.; LEITE, M. C. L.; CARNEIRO, R. G.; COLLEY, D.; GAZINELLI, G. \& KATZ, N., 1991. A multivariate analysis of socio-demographic factors, water contact and Schistosoma mansoni infection in an endemic arca in Brasil. Revista do Instituto de Medicina Tropical de São Paulo, 33: 58-63.

PAULINI, E. \& DIAS, E. P., 1971. Resultados de três anos de controle da esquistossomose em Belo Horizonte (MG). Revista Brasileira de Malariologia, 24: 151-172.

PELLEGRINO, J. \& KATZ, N., 1968. Experimental chemotherapy of Schistosomiasis mansom. Advances in Parasitolgy, 6: 223-290.

PESSOA, S. B. \& BARROS, P. R., 1953. Notas sobre a epidemiologia da esquistossomose mansônica no Estado de Sergipe. Revista de Medicina $e$ Cirurgia, 13: 17-24.

SANTOS, M. L. \& COURA, J. R., 1986. Morbidade da esquistossomose no Brasil. IV - Evolução em pacientes tratados e seus controles. Memórias do Instituto Oswaldo Cruz, 81: 53-60.

SCHLESSELMAN, J. J., 1982. Case Control Studies: Design, Conduct, Analysis. New York: Oxford University Press.

SNEDECOR, G. W. \& COCHRAN, W. G., 1977. Statistical Methods. Iow: State University Press.

WHO (World Health Organization), 1985. Schistosomiais Control. Geneve: WHO. (Technical Series, 728) 